\title{
Support vector regression is an improvement for principle component analysis
}

\author{
Mohamed, A. M. ${ }^{1} \quad$ Abdel Latif, S. H. ${ }^{2} \quad$ Alwan, A. S. ${ }^{3^{*}}$ \\ ${ }^{1}$ Faculty of Graduate Studies for Statistical Research, Cairo University, Cairo, Egypt \\ ${ }^{2}$ Faculty of Graduate Studies for Statistical Research,Cairo University, Cairo, Egypt \\ ${ }^{3}$ College of Administration \& Economics, Sulaimani University, Sulaimani , Iraq \\ 1dramanymousa04@gmail.com, ${ }^{2}$ shereen_hamdy_@hotmail.com \\ ${ }^{3 *}$ asraa.alwan@univsul.edu.iq
}

\begin{abstract}
:
The principle component analysis is used more frequently as variables reduction technique. And recently, an evolving group of studies make use of machine learning regression algorithms to improve the estimation of empirical models. One of the most frequently used machine learning regression model is support vector regression with various kernel functions. However, ensemble of support vector regression and principal component analysis is also possible. So, this paper aims to investigate the competence of support vector regression techniques after performing principal component analysis to explore the possibility of reducing data and having more accurate estimations. Some new proposals are introduced and the behavior of two different models $\varepsilon$-SVR and $v$-SVR are compared through an extensive simulation study under four different kernel functions; linear, radial, polynomial, and sigmoid kernel functions, with different sample sizes ranges from small, moderate to large. The models are compared with their counterparts in terms of coefficient of determination $\left(R^{2}\right)$ and root mean squared error (RMSE).The comparative results show that applying SVR after PCA models improve the results in terms of SV numbers between $30 \%$ and $60 \%$ in average and it can be applied with real data. In addition, linear kernel function gave the best values rather than other kernel functions and the sigmoidkernel gave the worst values. Under $\varepsilon$-SVR the results improved which did not happen with $v$-SVR. It is also drawn that, RMSE valuesdecreased with increasing sample size.
\end{abstract}

Keywords: $\varepsilon$-Support Vector Regression, Kernel Functions, Principal Component Analysis, $\mathcal{V}$ -Support Vector Regression.

\section{Introduction}

The recent trends in collecting huge and diverse datasets,such as documents, videos and digital images, financial time series, and gene expressions and DNA copy numbers,have posed a great challenge that is brought by the 
high dimensionality and aggravated by the presence of irrelevant dimensions in tasks such as predictive modeling[3].

PCAhelps in building a predictive model that is simple as it contains the smallest number of variables and efficient that accounts for as much of the information "explained variation” as possible[11],[6],[7],[12],[13].

PCA can be widely applied in all forms of analysis from neuroscience tocomputer graphics and in a variety of real-world applications including image segmentation [15], climate research [8], genome-wide expression studies [14], and deep learning [2] due to its superior properties, such as linear un-correlation, low-dimensionality and visualization in multivariate data, over other linear dimension reduction (LDR) methods[10],[13].

Support Vector Machine (SVM) is one of the most robust prediction methods, based on the statistical learning framework or VC theory proposed by Vapnik and Chervonenkis (1974) and Vapnik (1982, 1995).SVM seeks to maximize the predictive accuracy from computation of a confidence interval for the importance of a variable in order to describe the relationship between inputs and outputs[10]. SVMis a supervisedlearning model, with associated learning algorithm that analyze data usedfor classification, known as SV classifier, and regression (function approximation), known as support vector regression (SVR) [16].

During past few decades, an extension to the SVM classification algorithmhas been received a considerable attention, see [1], which is mainly due to VladimirVapnik and coworkers Harris Drucker, Christopher J. C. Burges, Linda Kaufman and Alexander J. SmolaIn 1996 for introducingSVM for regression, known as $\varepsilon$-SVR model,that handles regression problems [6]. SVR has additional advantages compared to other regression methods, see[16].

PCAis a widely applied feature extraction method in the framework of SVR. In the literature,[4]proposed an integration of PCA and SVR, or PCA-SVR,toenhance the performance of prediction (forecasting) model for financial time series. PCA-SVR produced less mean average precision MAP(\%), mean absolute error (MAE), root mean square error (RMSE) and mean square error (MSE) than single SVR, [16]proposed PCA-SVM stock selection model which achieves the entire accuracy of $75.44 \%$ in training set and of $61.79 \%$ in testing set.

Two types of procedures have been adopted within the practical aspect. The first procedure is applying the PCA within $\varepsilon-S V R$. The other is also applying the PCA but within $v-S V R$.

The rest of the paper is organized as follows: Section 2 presents themethodology thatused in this paper PCA and SVR. Section 3discussed the results and evaluation. Finally, conclusion and future work are given in Section 4.

\section{Methodology}

The data used in the PCA were recorded from a Monte Carlo simulationtechnique. The simulation was designed in the R software using R package. 5000 simulations with 1000 observations were carried out. The data followingnormal distribution. Eight sample sizes (50, $100,250,500,1000,1750$, and 2000) were used in this study. The dataset is 9-dimensional with 9 variables giving 9 principal components.Several analyses with two, three, and four 
features were performed to obtain the effective parameters on the fragmentation; these parameters were selectedas input parameters for the predictive models.

In this study, two main procedures were conducted on the same dataset. The first procedure was preceded in two steps: First, PCA was performed on a set of parameters- input (features) and output to estimate how far the effect of PCA on several sample sizes by calculatingrootmean square error (RMSE). Second, $\varepsilon-$ SVR was performed to show its effect, using a set of mathematical functions known as positive definite kernels- linear, polynomial, radial, and sigmoid. The second procedure followed the same steps of the first procedure but applying $v-S V R$ instead of $\varepsilon$-SVR. The next sections will cover all of these methods.

\subsection{Principal Component Analysis (PCA)}

Many raw data sets have a high-dimension space, and are accordingly difficult to interpret. In addressing this issue, PCA finds smaller number of uncorrelated components from high dimensional original inputs by calculating the eigenvectors of the covariance matrix. Given a set of $m$ dimensional input vectors $x_{i}=\left(x_{i}(1), x_{i}(2), \ldots, x_{i}(m)\right)^{T}$ where $i=1,2, \ldots, n$. PCA is a transformation of $x_{i}$ into a new vector $y_{i}$ by:

$y_{i}=U^{T} x_{i}(1)$

where $U$ is the $m \times$ morthogonal matrix whosejth column $u_{j}$ is the $j$ th eigenvector of

the sample covariance matrix $C=\frac{1}{n} \sum_{i=1}^{n} x_{i} x_{i}^{T}$. In other words, PCA solves the following eigenvalue problem:

$$
\lambda_{j} u_{j}=C u_{j}, j=1,2, \ldots, m
$$

where $\lambda_{j}$ is one of the eigenvalues of $C$ and $u_{j}$ is the corresponding eigenvector.

Based on the estimated $u_{j}$, the components of $y_{i}$ are calculated as the orthogonal transformation of $x_{i}$. That is,

$$
y_{i}(j)=u_{j}^{T} x_{i}, j=1,2, \ldots, m(3)
$$

The new components are called principal components. By using only the first several eigenvectors sorted in adescending order of eigenvalues, the number of principal components in $y_{i}$ can be reduced [11]. Thus, PCA can be used to reduce dimensions where the principal components are uncorrelated and have sequentially maximum variances.

\subsection{Support Vector Regression (SVR)}

The SVR extends the basic principles of SVM for classification [5] by measuring the error of approximation instead of the margin used in classification. SVR estimates a continuousvalued function that encodes the fundamental interrelation between a given input and its corresponding output in the training data. This function then can be used to predict outputs for given inputs that were not included in the training set. This is similar to a neural network. However, a neural network's solution is based on empirical risk minimization. In contrast, SVR introduces structural risk minimization into the regression and thereby achieves a global optimization, while a neural network achieves only a local minimum. Brief descriptions of two types of SVR which have been considered in the paper are given. 


\subsection{1. $\varepsilon$-SVR Model}

$\varepsilon$-SVR maps the input vectors $x_{i} \in R^{m}$ into a high dimensional feature space. Given a training set $\left(x_{i}, y_{i}\right), i=1,2, \ldots, n$, where $x_{i} \in R^{m}$ is the $m$-dimensional input vector and $y_{i} \in R$ is the response variable. SVR generates the linear regression function in the form of generic cost estimation model that can be written as

$y=f(x)=w, x+b=w^{T} x+b(4)$

wherew is the weight vector corresponding to $x$ and $b$ isthe bias. The Vapnik's lineare Insensitivity loss (error) function is also given as

$L(y, f(x))=\left\{\begin{array}{cc}0 & \text { if }|y-f(x)| \leq \varepsilon \\ |y-f(x)|-\varepsilon & \text { otherwise }\end{array}\right.$

Based on the above, the linear regression $f(x)$ is estimated by simultaneously minimizing $\|w\|^{2}$ and the sum of the linear $\varepsilon$-Insensitivity losses as shown in Equation (7).The constant $c$ controls a trade-off between an approximation error and the weight vector norm $w$ is a design parameter chosen by the user.

$R=\frac{1}{2}\|w\|^{2}+c\left(\sum_{i=1}^{n}|y-f(x)|_{\varepsilon}\right)$

Minimizing the risk $\mathrm{R}$ is equivalent to minimizing the following risk under the following constraints mentioned in Equations

$$
\begin{aligned}
& \text { Minimize } R=\frac{1}{2}\|w\|^{2}+c \sum_{i=1}^{n}\left(\xi_{i}+\xi_{i}^{*}\right)(7) \\
& \text { subject to }\left\{\begin{array}{c}
\left(w^{T} x_{i}+b\right)-y_{i} \leq \varepsilon+\xi_{i} \\
y_{i}-\left(w^{T} x_{i}+b\right) \leq \varepsilon+\xi_{i}^{*} \\
\xi_{i}, \xi_{i}^{*} \geq 0, i=1,2, \ldots, m
\end{array}\right.
\end{aligned}
$$

Here, $\xi_{i}$ and $\xi_{i}^{*}$ are slack variables, one for exceeding the target value by more than $\varepsilon$ and other for being more than $\varepsilon$ below the target. As used in SVM, the above constrained optimization problem is solved using Lagrangian theory and the Karush-Kuhn-Tucker (KKT) conditions for the optimum of a constrained functionto obtain the desired weight vector of the regression function[17].

In Equation (4), the generalization performance of such linearfunction, $f(x)$, is fairly limited and unable to reflect thetrue regression procedure. In order to overcome suchweakness, a standard mathematical solution is the introductionof kernel function, $\varphi(X)$, which is a nonlinearmapping function from the input space to a higher dimensionalfeature space. We can reachinfinite dimensions for a more expressive $f$ by using $\varphi(X)$. The most popular kernel functions used in this study areshownin Table (2.1).

Table (2.1) Admissible kernel functions

\begin{tabular}{|l|c|c|}
\hline Name & Definition & Parameter \\
\hline Linear & $k\left(x_{1}, x_{2}\right)=x_{1} \cdot x_{2}$ & - \\
\hline Polynomial & $k\left(x_{i}, x_{j}\right)=\left(x_{i} \cdot x_{j}+1\right)^{d}$ & $d$ \\
\hline Radial basis function & $k\left(x_{i}, x_{j}\right)=\exp \left(-\gamma\left\|x_{i}-x_{j}\right\|^{2}\right)$ & $\gamma$ \\
\hline Sigmoid & $k(x, y)=\tanh \left(\alpha x^{T} y+c\right)$ & $c$ \\
\hline
\end{tabular}




\subsection{2. $v$-SVR Model}

$v$-SVR is one of the most popular modifications proposed by Scho"lkopf, Bartlett, Smola, and Williamson (1999). The benefit of $v$-SVR is that it provides a way to automatically minimize $\varepsilon$.In $\varepsilon$-SVR, selection of a proper $\varepsilon$ value is essential for an accurate regression approximation. However, it is difficult to specify $\varepsilon$ beforehand, other than an empirical choice. In $v$-SVR a new parameter of a prior $v \in(0,1)$ is introduced to automatically adjust a flexible tube by controlling the number of support vector and tolerated training errors. Then, the parameter $\varepsilon$ becomes a variable in the optimization process and is controlled by the new parameter $v$.In $v$-SVR, the optimization problem can be written, given a function $\varphi(x)$ to the kernel space for a nonlinear case, as follows

$$
\begin{gathered}
\min _{w} \frac{1}{2}\|w\|^{2}+C\left(v \varepsilon+\frac{1}{l} \sum_{i=1}^{l}\left(\xi+\xi^{*}\right)\right) \\
\text { subjectto }\left\{\begin{array}{c}
y_{i}-w^{T} \varphi\left(x_{i}\right)-b \leq \varepsilon+\xi \\
w^{T} \varphi\left(x_{i}\right)+b-y_{i} \leq \varepsilon+\xi^{*}(9) \\
\xi, \xi^{*}, \varepsilon \geq 0
\end{array}\right.
\end{gathered}
$$

Here, the newly introduced constant variable $v \in(0,1)$ is used as atrade-off against model complexity and slack variables. Forming aLagrangian formulation from $\left(\varphi()=.R^{d} \rightarrow F\right)$ by introducing positive multipliers $\alpha, \alpha^{*}, \eta, \eta^{*}$ and $b$ gives

$$
\begin{aligned}
& L\left(w, \xi, \xi^{*}, \alpha, \alpha^{*}, \eta, \eta^{*}, \beta\right)=\frac{1}{2}\|w\|^{2}+C v \varepsilon+\frac{C}{l} \sum_{i=1}^{l}\left(\xi_{i}+\xi_{i}^{*}\right) \\
& +\sum_{i=1}^{l} \alpha_{i}^{*}\left(y_{i}-w^{T} x_{i}-b-\varepsilon-\xi_{i}\right)+\sum_{i=1}^{l} \alpha_{i}\left(w^{T} x_{i}+b-y_{i}-\varepsilon-\xi_{i}^{*}\right) \\
& -\sum_{i=1}^{l}\left(\eta_{i} \xi_{i}+\eta_{i}^{*} \xi_{i}^{*}\right) \beta \varepsilon(10)
\end{aligned}
$$

Following the KKT conditions that partial derivatives with respect tothe variables $w, b, x, x^{*}$, and $\varepsilon$ are equal to be zero and the products of theLagrange multipliers and the constraint are equal to zero, we have thefollowing dual optimization problem of $v$-SVR

$\max _{\alpha, \alpha^{*}} \sum_{l}^{l_{s v}} y_{i}\left(\alpha_{i}-\alpha_{i}^{*}\right)-\frac{1}{2} \sum_{i}^{l_{s v}} \sum_{j}^{l_{s v}}\left(\alpha_{i}-\alpha_{i}^{*}\right)\left(\alpha_{j}-\alpha_{j}^{*}\right) k\left(x_{i}, x_{j}\right)$,

where $k\left(x_{i}, x_{j}\right)=\varphi\left(x_{i}\right) \varphi\left(x_{j}\right)$ subject to

$\sum_{i}^{l_{s v}}\left(\alpha_{i}-\alpha_{i}^{*}\right)=0, \alpha_{i}, \alpha_{i}^{*} \in\left[0, \frac{C}{l}\right], \sum_{i}^{l_{s v}}\left(\alpha_{i}-\alpha_{i}^{*}\right) \leq C v(11)$

Then, the regression estimate takes the form

$f(x)=\sum_{i}^{l_{s v}}\left(\alpha_{i}-\alpha_{i}^{*}\right) k\left(x_{i}, x\right)+b(12)$

Compared to the optimization problem in $\varepsilon$-SVR [10], we can see thatthe parameter $\varepsilon$ vanishes but instead there is the new parameter $v$ in $v$-SVR [9]. Scho"lkopf et al. had proved that $v \in(0,1)$ is an upper bound on the fraction of errors (i.e., data points outside of the tube divided by the total number of data points $l$ ) and a lower bound on the fraction of support vectors (i.e., the numbers of support vectors divided by the total number of data points $l$ ). 


\section{Simulation Study: Results and Discussion}

This simulation was conducted for the purpose of comparison between $\varepsilon$-SVR and $v$ SVR models after and before applying PCA using four different kernel functions to detect the PCA effect on data reduction. In this study, all trained models designed are evaluated using measured data based on two different measures, root mean square error (RMSE), and coefficient of determination $\left(R^{2}\right)$. RMSE is a commonly used measure of the difference between predicted values of model and the actual values from the system that is being modeled. The sample sizes are arbitrarily determined to represent small, moderate, and large sample sizes such as; $n=50,100,250,500,1000,1500,1750$, and 2000. The simulation results were based on10000 replications. All computations are using the R program (R x64 3.2.5) version.

Two types of procedures have been adopted within the practical aspect.The first procedure lays in studying and applying the data reduction method, PCA, within $\varepsilon$-SVR model with four different kernel functions; linear, polynomial, redial, sigmoid, which was referred to previously through which we can use this to simulate a large number of hypothetical cases that may arise within the practical aspect. Thus, generalizing the results becomes more comprehensive. According to the importance of the components, table (a) [SeeAppendix (a)], show that components one and two together capture from $86 \%$ to $95 \%$.

\subsection{Effect on Support Vector Regression Number:}

Tables from (b.1) to (b.8)[See Appendix (b)], show that before using PCA, the $\varepsilon$-SVR application reduced the number of SVR by $30 \%$ to $40 \%$, with the linear kernel being superior to its counterparts at most sample sizes, and it reached $70 \%$ for sample size 100 , and the polynomial kernel showed good performance with large sample sizes 1750 and 2000.But the $v$-SVR application reduced the number of SVR by a rate ranging from $53 \%$ to $60 \%$ with the sigmoid kernel function being superior to its counterparts at all most all sample sizes, the performance of all functions converged.And after applying PCA with $\varepsilon$-SVR, the percentage was about $30 \%$ with the radial kernel function superior to its counterparts with all most all sample sizes, and only in one case the decrease in the number of SVR reached $56 \%$ at $n=1750$. But with $v$-SVR, the results of sigmoid and linear kernel functions were equal for $50 \%$ of the cases, and the percentage ranged from $54 \%$ to $60 \%$, and the differences between them and polynomial were very narrowfollowed by the radial kernel function.

\subsection{Effect of Sample Size}

The following two figures illustrated the effect of sample size on the RMSE values. 


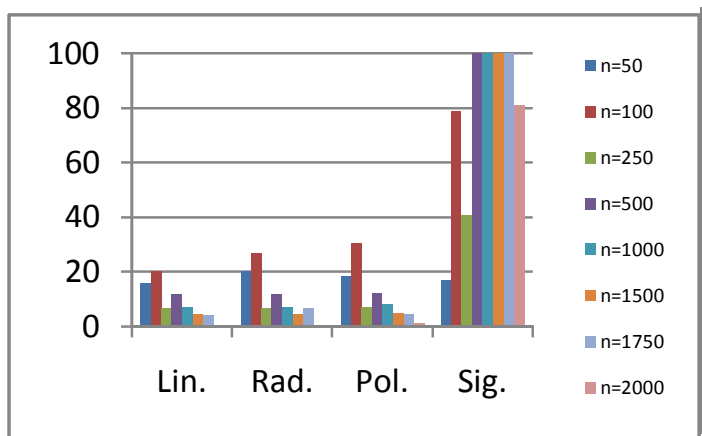

Figure (3.1) the effects of $n$ under $\varepsilon$-SVR before using PCA

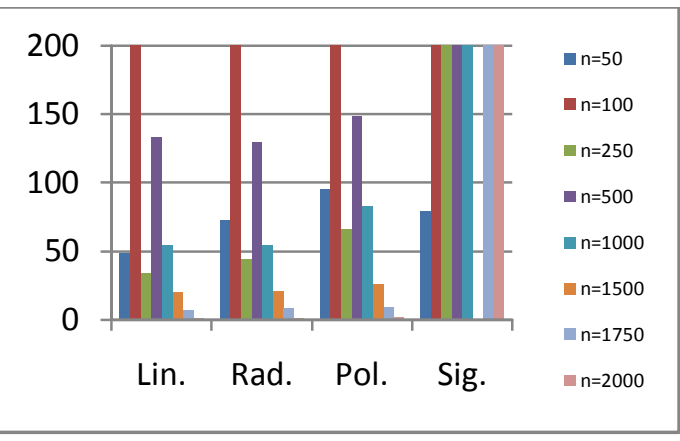

Figure (3.2) the effects of $n$ under $v$-SVR before using PCA

Under $\varepsilon$-SVR and $v$-SVR and with all sample sizes from $n=50$ to $n=2000$, after using PCA, and from figures (3.1) and (3.2), it is clear that the root mean square error with almost different kernel functionsdecreased with increasing sample size except for sample size $n=100$ and $n=500$. But the worst values of RMSE were at $n=100$ and $n=500$.A sample size $n=250$ gave good results for linear, radial, and polynomial kernel functions between sample sizes of 50 to 1000. [See Appendix (c), figures (c.1) and (c.2) for before using PCA case]

\subsection{Effect of PCA}

The following figures show the effects PCA with small, moderate, and large sample size under $\varepsilon$ SVRbefore and after using PCA.

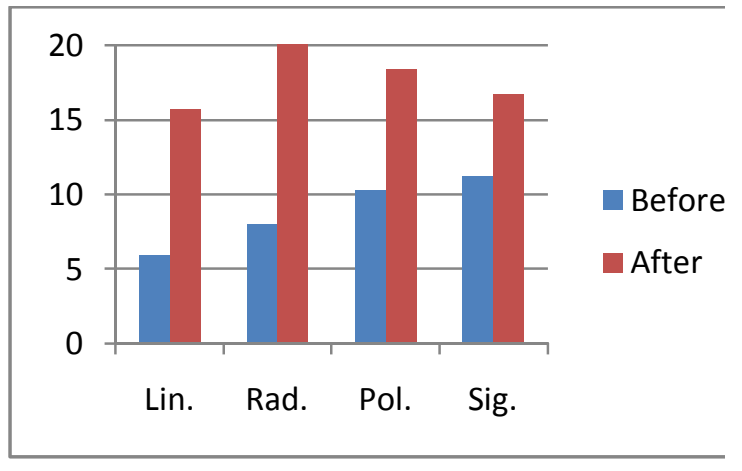

Fig. (3.3) effect of PCA for $\varepsilon$-SVRat $n=50$

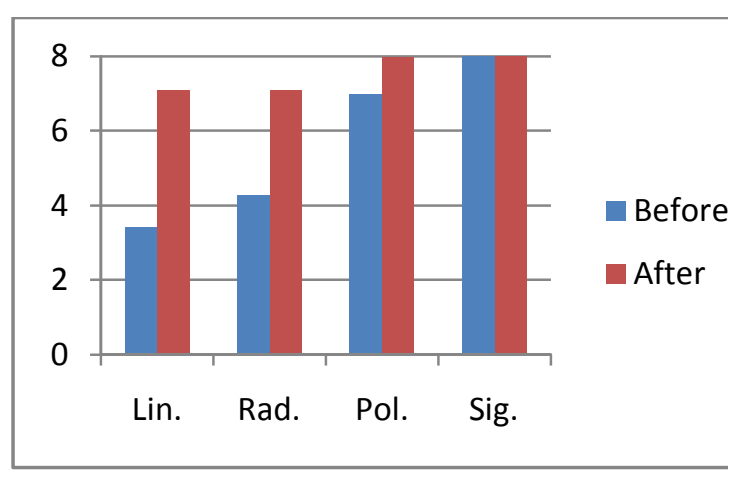

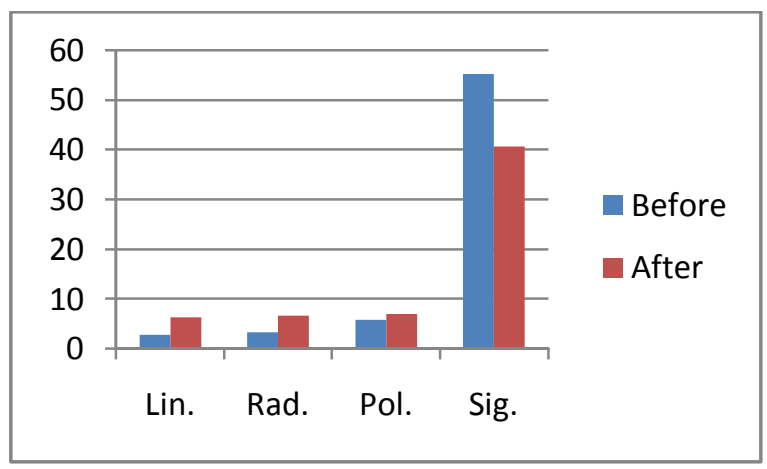

Fig. (3.4) effect of PCA for $\varepsilon$-SVR at $n=250$

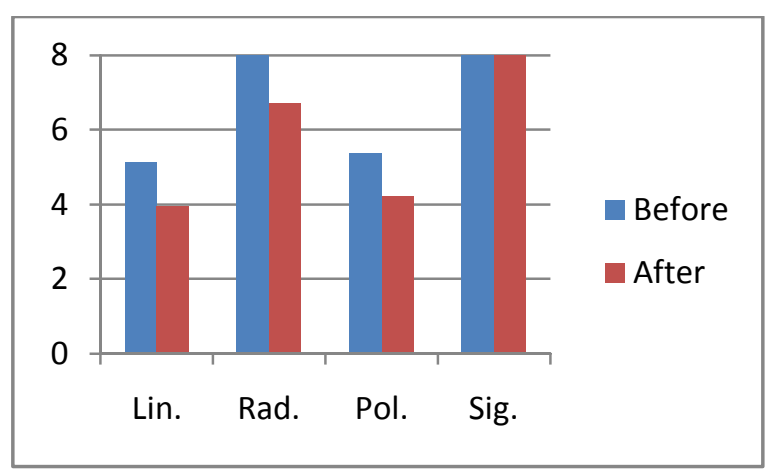


Fig. (3.5) effect ofPCA for $\varepsilon$-SVRat $n=1000$

Fig. (3.6) effect of PCA for $\varepsilon$-SVRat $n=1750$

Under $\varepsilon$-SVR, and with sample size $n=50,250,1000,1750$, before and after using PCA, and from figure (3.3), figure (3.4), figure (3.5) and figure (3.6) respectively, it can be concluded that for sample size greater than or equal 1500, results improved after applying PCA. And, it is clear that the root mean square error with linear kernel function gave the best values other than other kernel functions. In addition, the sigmoidkernel gave the worst values for RMSE amongst the other kernel functions.[See Appendix(c), figures from (c.3) to (c.6) for $n=100,500,1500,2000]$. The following figures show the effect of PCA under $v$-SVR.

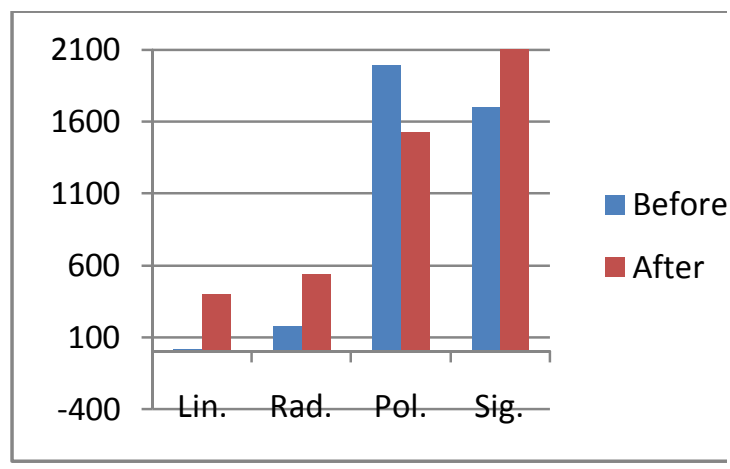

Fig. (3.7) effect of PCA for $v$-SVRat $n=100$

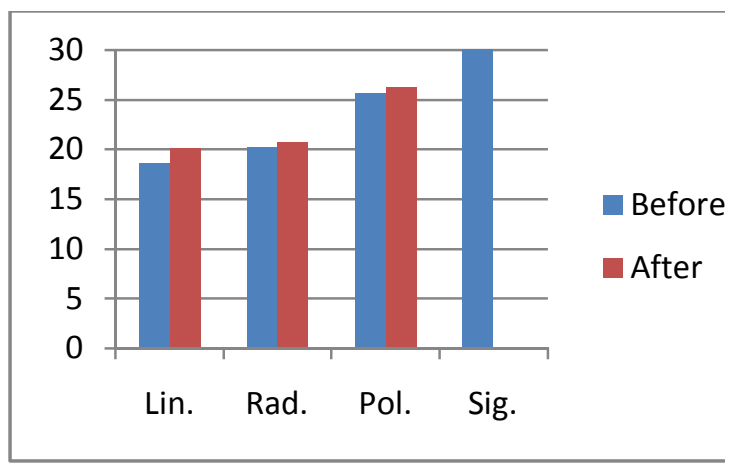

Fig. (3.9) Effect of PCA for $v$-SVR at $n=$ 1500

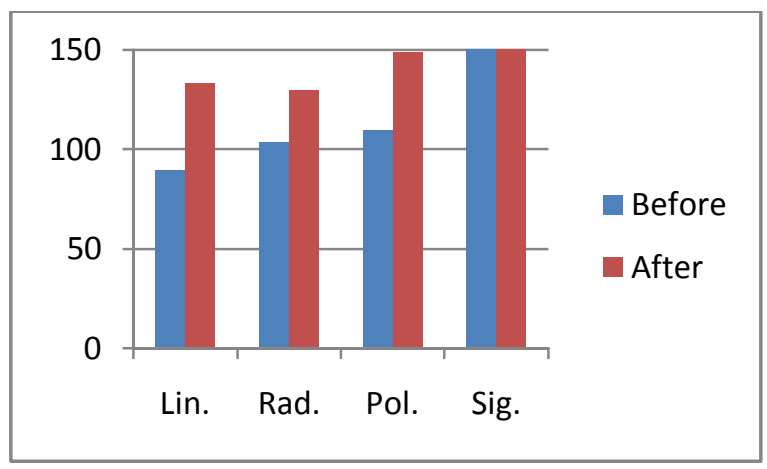

Fig. (3.8) effect of PCA for $v$-SVR at $n=500$

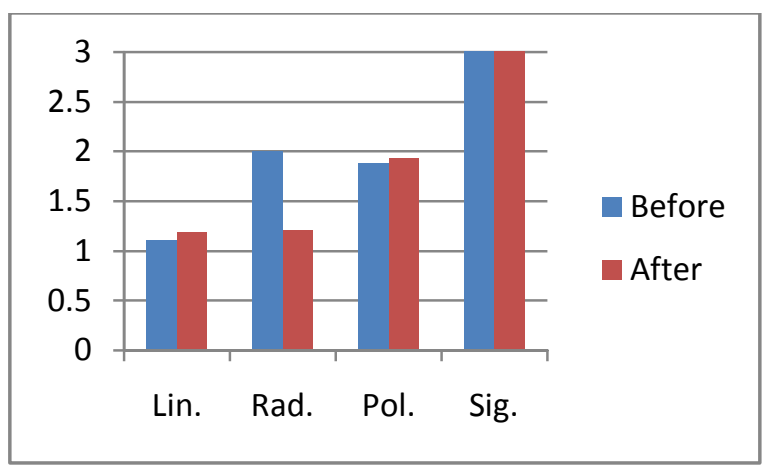

Fig. (3.10) Effect of PCA forv-SVRat $n=2000$

As it can be seen, under $v$-SVR, and with sample size $n=100,500,1500,2000$, from figure (3.7) to figure (3.10), it can be concluded that the $v$-SVR results did not improve after applying PCA with all sample sizes.Kernel function gave the best values rather than other kernel functions. In addition, the sigmoidkernel gave the worst values for RMSE amongst the other kernel functions. [See Appendix (c), figures from (c.7) to (c.10) forn $=50,250,1000,1750]$

\subsection{Effect of Support Vector Regression}

The following figures showed thatwith all types of kernel functions and at all sample sizes $\mathcal{E}$ SVR is better than $v$-SVR after using PCA, except for only one case for $n=1500$ and 
sigmoid. These results were similar to results obtained before using PCA [See Appendix (c), figures from (3.11) to(3.14)].

Figure (3.7) the effects of $\varepsilon$-SVR and $v$-SVR with all kernels functions, and for all sample sizes,after using PCA

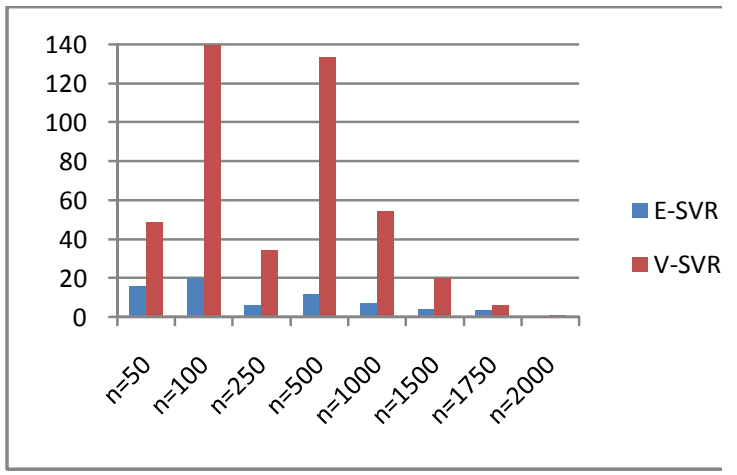

Fig. (3.11) Effect of SVR for Linear kernel

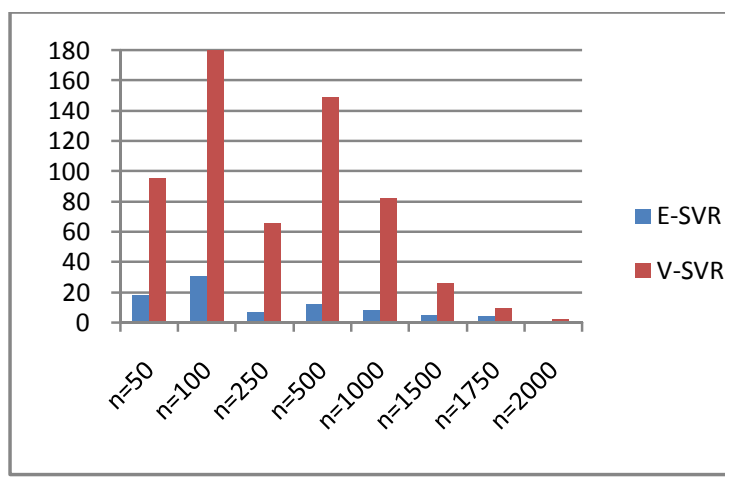

Fig. (3.13) Effect of SVR for Polynomial kernel

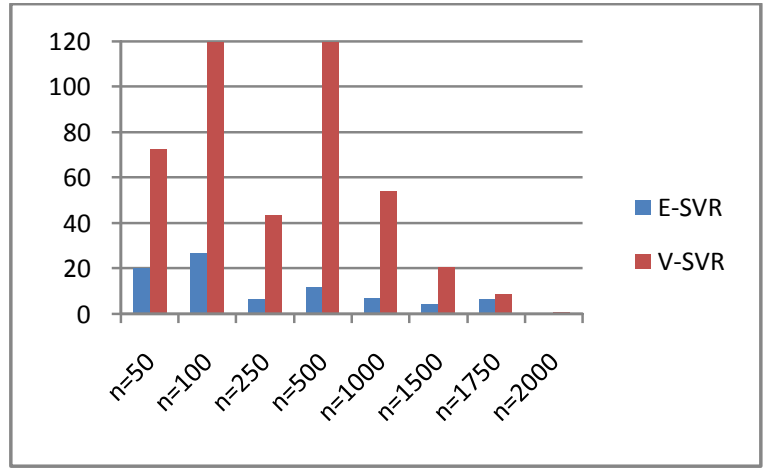

Fig. (3.12) Effect of SVR for Radial kernel

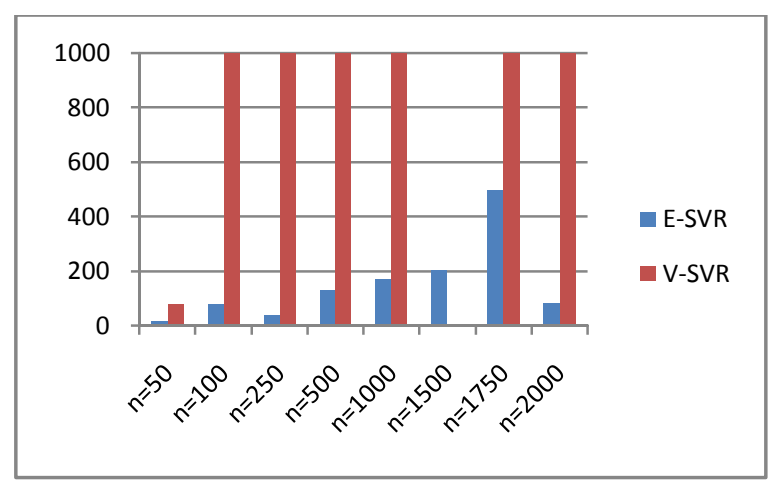

Fig. (3.14) Effect of SVR for Sigmoid kernel

\subsection{Effect of kernel function}

The following to figures (3.15) and (3.16) illustrates the effects of kernel function on RMSE with $\varepsilon$-SVR and $v$-SVR, at all sample sizes, after using PCA.

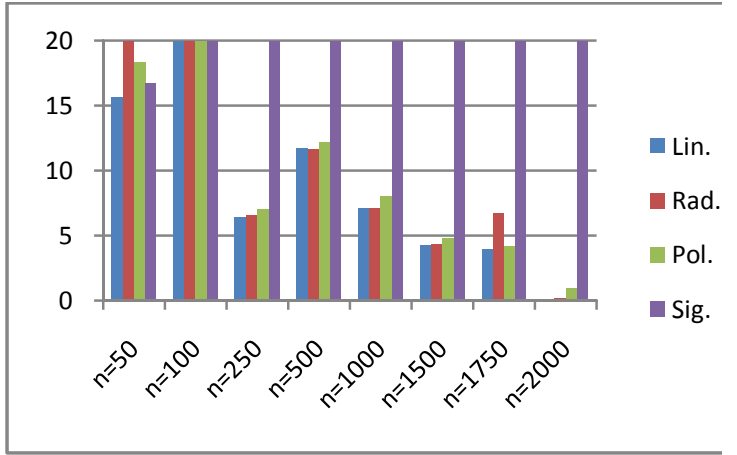

Fig. (3.15)E-SVR after PCA

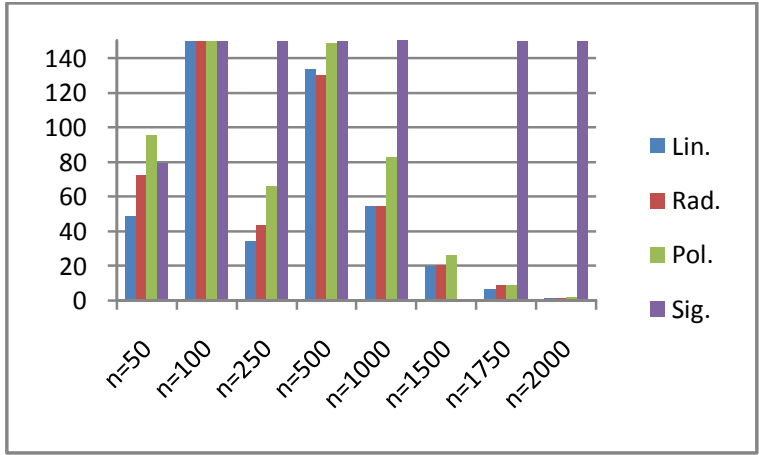

Fig. (3.16) $v$-SVR after PCA

Under $\varepsilon$-SVR, and for all sample sizes from $n=50$ to $n=2000$, after using PCA, and from figure (3.15), it is clear that the linear kernel function gave the best of root mean square error then the radial kernel function for all sample sizes except at $n=50$, it gave the worst 
value. But the sigmoid kernel function gave the worst value of RMSE with all sample sizes (except at $n=50$ ). It is worth noting that with the increase in the sample size, the results of linear, radial and polynomial are close to each other, except for radial when the sample size $n=1750$. The results of $v$-SVR are very similar to the results of $\varepsilon$-SVR except for sample size $n=100$ the polynomial kernel function gave the worst error value, and for sample sizes $n=500$ the radial kernel function gave the best error value, figure (3.16). These results are similar to results before using PCA. [See Appendix (c), figures (3.15) and (3.16)].

\section{General Conclusions}

When reducing the data dimensions, it's important not to lose more information than is necessary. Principal Component Analysis (PCA) is a well-established mathematical technique for reducing the dimensionality of data, while keeping as much variation as possible as we notes in practical section. It is also known that using of SVR with various kernel functions improves the estimation of models. The behavior of two different models $\varepsilon$-SVR and $v$-SVR are compared through an extensive simulation study under four different kernel functions; linear, radial, polynomial, and sigmoid kernel functions, with different sample sizes ranges from small, moderate to large. Generally,it can be concluded that according to the reduction of SVR, after applying PCA and with all sample sizes, under $\varepsilon$-SVR, the percentage of reduction was about $30 \%$ with the radial kernel function. But underv-SVR, the result of sigmoid and linear kernel functions were the best between other counterparts, and the percentage ranged from $54 \%$ to $60 \%$. But with regard to the value of RMSE, under $\varepsilon$-SVR, for sample size greater than or equal 1500, results improved. And, it is clear that the RMSE with linear kernel function gave the best values rather than other kernel functions. In addition, the sigmoidkernel gave the worst values amongst the other kernel functions. But under $v$-SVR, results did not improve after applying PCA with all sample sizes. It is also drawn that, with $\varepsilon$ SVR and $v$-SVR, the RMSE with almost different kernel functionsdecreased with increasing sample size which is considered as an indicator to the consistency. In addition, from sample sizes ranges from $n=50$ to $n=1000$, the sample size $n=250$ gave good results for linear, radial, and polynomial kernel functions.

\section{References}

1. Boser, Bernhard E.; Guyon, Isabelle M.; Vapnik, Vladimir N. (1992). "A training algorithm for optimal margin classifiers". Proceedings of the fifth annual workshop on Computational learning theory '92.p. 144. CiteSeerX 10.1.1.21.3818. doi:10.1145/130385.130401. ISBN 978897914970. S2 CID 207165665

2. Chan, T. H., Jia, K., Gao, S., Lu, J., Zeng, Z., \& Ma, Y. (2015). PCANet: A simple deep learning baseline for image classification?. IEEE transactions on image processing, 24(12), 5017-5032. 
3. Chao, D., Zhou, W., Ye, C., Zhang, Q., Chen, Y., Gu, L., ...\&Qiao, S. Z. (2019). An Electrolytic Zn-MnO2 Battery for High-Voltage and Scalable Energy Storage. AngewandteChemie International Edition, 58(23), 7823-7828.

4. Chowdhury, U. N., Chakravarty, S. K., \&Hossain, M. T. (2018). Short-term financial time series forecasting integrating principal component analysis and independent component analysis with support vector regression. Journal of Computer and Communications, 6(03), 51.

5. Cortes, C. and Vapnik, V. (1995) Support-Vector Networks. Machine Learning, 20,273-297. https://doi.org/10.1007/BF00994018

6. Engelbrecht, A. P. (2007). Computational intelligence: an introduction. John Wiley \& Sons.

7. Glaser, J. I., Benjamin, A. S., Farhoodi, R., \&Kording, K. P. (2019). The roles of supervised machine learning in systems neuroscience. Progress in neurobiology, 175, 126-137.

8. Hannachi, A., Jolliffe, I. T., \& Stephenson, D. B. (2007). Empirical orthogonal functions and related techniques in atmospheric science: A review. International Journal of Climatology: A Journal of the Royal Meteorological Society, 27(9), 1119-1152.

9. Higuchi, I., \&Eguchi, S. (2004). Robust principal component analysis with adaptive selection for tuning parameters. Journal of Machine Learning Research, 5(May), 453-471.

10. Hua, X. G., Ni, Y. Q., Ko, J. M., \& Wong, K. Y. (2007). Modeling of temperature-frequency correlation using combined principal component analysis and support vector regression technique. Journal of Computing in Civil Engineering, 21(2), 122-135.

11. Jolliffe, I. T. (1986). Principal components in regression analysis. In Principal component analysis (pp. 129-155). Springer, New York, NY.

12. Jolliffe, I. T., \&Cadima, J. (2016). Principal component analysis: a review and recent developments. Philosophical Transactions of the Royal Society A: Mathematical, Physical and Engineering Sciences, 374(2065), 20150202.

13. Lee, J. A., \&Verleysen, M. (2009). Quality assessment of dimensionality reduction: Rankbased criteria. Neurocomputing, 72(7-9), 1431-1443.

14. Ringnér, M. (2008). What is principal component analysis?. Nature biotechnology, 26(3), 303-304.

15. Shi, S., Li, G., Chen, H., Hu, Y., Wang, X., Guo, Y., \& Sun, S. (2018). An efficient VRF system fault diagnosis strategy for refrigerant charge amount based on PCA and dual neural network model. Applied Thermal Engineering, 129, 1252-1262.

16. Zhang, F., \& O'Donnell, L. J. (2020). Support vector regression. In Machine Learning (pp. 123-140). Academic Press.

17. Zhang, Y., d'Aspremont, A., \& El Ghaoui, L. (2012). Sparse PCA: Convex relaxations, algorithms and applications. In Handbook on Semidefinite, Conic and Polynomial Optimization (pp. 915-940). Springer, Boston, MA. 


\section{Appendix (a)}

Table (a): Evaluation of componentsfor all sample size

\begin{tabular}{|c|c|c|c|c|c|c|}
\hline & $\mathrm{n}$ & Comp.1 & Comp.2 & Comp.3 & Comp.4 & Comp.5 \\
\hline Standard deviation & \multirow{3}{*}{50} & 2.399582 & 1.493121 & 0.850262 & 0.466969 & 0.263411 \\
\hline Proportion of variance & & 0.639777 & 0.247712 & 0.080327 & 0.024229 & 0.00771 \\
\hline Cumulative Proportion & & 0.639777 & 0.887489 & 0.967817 & 0.992046 & 0.999755 \\
\hline Standard deviation & \multirow{3}{*}{100} & 2.405992 & 1.406846 & 0.941638 & 0.540262 & 0.194417 \\
\hline Proportion of variance & & 0.6432 & 0.219913 & 0.09852 & 0.032431 & 0.0042 \\
\hline Cumulative Proportion & & 0.6432 & 0.863113 & 0.961633 & 0.994064 & 0.998264 \\
\hline Standard deviation & \multirow{3}{*}{250} & 2.4141047 & 1.6512802 & 0.49868025 & 0.43419256 & 0.08290096 \\
\hline Proportion of variance & & 0.6475446 & 0.3029696 & 0.02763133 & 0.02094702 & 0.00076361 \\
\hline Cumulative Proportion & & 0.6475446 & 0.9505142 & 0.97814554 & 0.99909256 & 0.99985618 \\
\hline Standard deviation & \multirow{3}{*}{500} & 2.251667 & 1.696724 & 0.999785 & 0.221098 & 0.051637 \\
\hline Proportion of variance & & 0.563334 & 0.319875 & 0.111063 & 0.005432 & 0.000296 \\
\hline Cumulative Proportion & & 0.563334 & 0.883208 & 0.994272 & 0.999703 & 0.999999 \\
\hline & & & & & & \\
\hline Standard deviation & \multirow{3}{*}{1000} & 2.124317 & 1.818052 & 0.977601 & 0.410422 & 0.209954 \\
\hline Proportion of variance & & 0.501414 & 0.367257 & 0.106189 & 0.018716 & 0.004898 \\
\hline Cumulative Proportion & & 0.501414 & 0.868671 & 0.97486 & 0.993576 & 0.998474 \\
\hline Standard deviation & \multirow{3}{*}{1500} & 2.637062 & 1.097019 & 0.91294 & 0.083799 & 0.036359 \\
\hline Proportion of variance & & 0.772678 & 0.133717 & 0.092607 & 0.00078 & 0.000147 \\
\hline Cumulative Proportion & & 0.772678 & 0.906394 & 0.999001 & 0.999781 & 0.999928 \\
\hline Standard deviation & \multirow{3}{*}{1750} & 2.581409 & 1.22201 & 0.699998 & 0.447875 & 0.374477 \\
\hline Proportion of variance & & 0.740408 & 0.165923 & 0.054444 & 0.022288 & 0.015581 \\
\hline Cumulative Proportion & & 0.740408 & 0.906331 & 0.960776 & 0.983063 & 0.998645 \\
\hline Standard deviation & \multirow{3}{*}{2000} & 2.601504 & 1.028339 & 0.904634 & 0.485987 & 0.333533 \\
\hline Proportion of variance & & 0.751981 & 0.117498 & 0.090929 & 0.026243 & 0.012361 \\
\hline Cumulative Proportion & & 0.751981 & 0.869479 & 0.960408 & 0.98665 & 0.999011 \\
\hline
\end{tabular}




\section{Appendix (b)}

Table (b.1):Application results of $\varepsilon$-SVR and $v$-SVR with $\mathrm{n}=50$

\begin{tabular}{|c|c|c|c|c|c|c|c|}
\hline \multirow{2}{*}{ Kernel } & Using & \multicolumn{3}{|c|}{$\varepsilon$-SVR=50 } & \multicolumn{3}{c|}{$v$-SVR=50 } \\
\cline { 3 - 8 } & PCA & No. SVR & RMSE & & No. SVR & RMSE & \\
\hline \multirow{2}{*}{ Linear } & Before & 35 & 5.905735 & 0.611466 & 26 & 4.487617 & 0.648434 \\
\cline { 2 - 8 } & After & 34 & 15.63424 & 0.920805 & 22 & 6.973119 & 0.922534 \\
\hline \multirow{2}{*}{ Polynomial } & Before & 41 & 10.24338 & 0.518209 & 31 & 10.64776 & 0.525611 \\
\cline { 2 - 8 } & After & 36 & 18.34726 & 0.824364 & 22 & 9.766403 & 0.820838 \\
\hline \multirow{2}{*}{ Radial } & Before & 37 & 7.983526 & 0.317025 & 30 & 10.44179 & 0.367461 \\
\cline { 2 - 8 } & After & 36 & 20.07923 & 0.109865 & 23 & 8.525746 & 0.150681 \\
\hline \multirow{2}{*}{ Sigmoid } & Before & 37 & 11.18416 & 0.783092 & 24 & 10.15318 & 0.752485 \\
\cline { 2 - 8 } & After & 40 & 16.73461 & 0.692276 & 20 & 8.911357 & 0.737407 \\
\hline
\end{tabular}

Table (b.2): Application of $\varepsilon$-SVR and $v$-SVR with $n=100$

\begin{tabular}{|c|c|c|c|c|c|c|c|}
\hline \multirow{2}{*}{ Kernel } & Using & \multicolumn{3}{|c|}{$\varepsilon$-SVR=100 } & \multicolumn{3}{c|}{$v$-SVR=100 } \\
\cline { 3 - 8 } & PCA & No. SVR & RMSE & $R^{2}$ & No. SVR & RMSE & $R^{2}$ \\
\hline \multirow{2}{*}{ Linear } & Before & 30 & 3.812108 & 0.989790 & 44 & 3.898366 & 0.989469 \\
\cline { 2 - 8 } & After & 70 & 20.12219 & 0.734026 & 42 & 19.94299 & 0.730193 \\
\hline \multirow{2}{*}{ Polynomial } & Before & 70 & 45.75615 & 0.618775 & 48 & 44.65717 & 0.584493 \\
\cline { 2 - 8 } & After & 72 & 30.64740 & 0.380796 & 43 & 38.99390 & 0.114258 \\
\hline \multirow{2}{*}{ Radial } & Before & 42 & 22.98349 & 0.645508 & 51 & 13.33926 & 0.644507 \\
\cline { 2 - 8 } & After & 70 & 26.87716 & 0.475720 & 46 & 23.15135 & 0.449809 \\
\hline \multirow{2}{*}{ Sigmoid } & Before & 80 & 74.41939 & 0.044944 & 41 & 41.14962 & 0.000311 \\
\cline { 2 - 8 } & After & 72 & 78.73017 & 0.105730 & 42 & 75.49266 & 0.107149 \\
\hline
\end{tabular}

Table (b.3): Application results of $\varepsilon$-SVR and $v$-SVR with $\mathrm{n}=250$

\begin{tabular}{|c|c|c|c|c|c|c|c|}
\hline \multirow{2}{*}{ Kernel } & Using & \multicolumn{3}{|c|}{$\varepsilon$-SVR=250 } & \multicolumn{3}{c|}{$v$-SVR=250 } \\
\cline { 3 - 8 } & PCA & No. SVR & RMSE & $R^{2}$ & No. SVR & RMSE & $R^{2}$ \\
\hline \multirow{2}{*}{ Linear } & Before & 149 & 2.7591288 & 0.896497 & 103 & 3.0581533 & 0.8846127 \\
\cline { 2 - 8 } & After & 179 & 6.4037621 & 0.44426 & 102 & 5.8314337 & 0.5741451 \\
\hline \multirow{2}{*}{ Polynomial } & Before & 184 & 5.87614584 & 0.613779 & 105 & 7.9868842 & 0.3640643 \\
\cline { 2 - 8 } & After & 182 & 7.0393593 & 0.35889 & 104 & 8.12225091 & 0.2861072 \\
\hline \multirow{2}{*}{ Radial } & Before & 156 & 3.3402499 & 0.858116 & 111 & 4.5304271 & 0.7494853 \\
\cline { 2 - 8 } & After & 176 & 6.6047535 & 0.40594 & 108 & 6.5998772 & 0.4548942 \\
\hline \multirow{2}{*}{ Sigmoid } & Before & 196 & 55.216609 & 0.001994 & 102 & 40.6345665 & 0.008469012 \\
\cline { 2 - 8 } & After & 199 & 40.581904 & 0.00173 & 102 & 38.9927044 & 0.0165334 \\
\hline
\end{tabular}

Table (b.4):Application results of $\varepsilon$-SVR and $v$-SVR with $n=500$

\begin{tabular}{|c|c|c|c|c|c|c|c|}
\hline \multirow{2}{*}{ Kernel } & Using & \multicolumn{3}{|c|}{$\varepsilon$-SVR=500 } & \multicolumn{3}{c|}{$v$-SVR=500 } \\
\cline { 3 - 8 } & PCA & No. SVR & RMSE & $R^{2}$ & No. SVR & RMSE & $R^{2}$ \\
\hline \multirow{2}{*}{ Linear } & Before & 361 & 9.975281 & 0.343531 & 203 & 9.466509 & 0.329342 \\
\cline { 2 - 8 } & After & 366 & 11.73772 & 0.098096 & 202 & 11.55629 & 0.329342 \\
\hline \multirow{2}{*}{ Polynomial } & Before & 360 & 11.99407 & 0.146126 & 205 & 10.463957 & 0.126680 \\
\cline { 2 - 8 } & After & 357 & 12.17787 & 0.02367 & 203 & 12.19348 & 0.1266801 \\
\hline \multirow{2}{*}{ Radial } & Before & 360 & 11.44221 & 0.152811 & 218 & 10.176497 & 0.146262 \\
\cline { 2 - 8 } & After & 368 & 11.65505 & 0.125827 & 214 & 11.388279 & 0.146262 \\
\hline
\end{tabular}




\begin{tabular}{|c|c|c|c|c|c|c|c|}
\hline \multirow{2}{*}{ Sigmoid } & Before & 399 & 122.0327 & 0.035544 & 202 & 102.1085 & 0.0025699 \\
\cline { 2 - 8 } & After & 394 & 130.80145 & 0.010471 & 201 & 90.1976 & 0.00256991 \\
\hline
\end{tabular}

Table (b.5): Application results of $\varepsilon$-SVR and $v$-SVR with $n=1000$

\begin{tabular}{|c|c|c|c|c|c|c|c|}
\hline \multirow{2}{*}{ Kernel } & Using & \multicolumn{3}{|c|}{$\varepsilon$-SVR=1000 } & \multicolumn{3}{c|}{$v$-SVR=1000 } \\
\cline { 3 - 8 } & PCA & No. SVR & RMSE & $R^{2}$ & No. SVR & RMSE & $R^{2}$ \\
\hline \multirow{2}{*}{ Linear } & Before & 618 & 3.42460 & 0.8826332 & 405 & 3.100595 & 0.882584 \\
\cline { 2 - 8 } & After & 720 & 7.08211 & 0.5049097 & 401 & 7.3756314 & 0.504739 \\
\hline \multirow{2}{*}{ Polynomial } & Before & 687 & 6.975845 & 0.560541 & 413 & 8.443815 & 0.547097 \\
\cline { 2 - 8 } & After & 721 & 7.98206 & 0.3652298 & 402 & 9.083121 & 0.350718 \\
\hline \multirow{2}{*}{ Radial } & Before & 636 & 4.2676199 & 0.825030 & 438 & 4.0220206 & 0.818858 \\
\cline { 2 - 8 } & After & 717 & 7.1008020 & 0.504075 & 413 & 7.3552450 & 0.501977 \\
\hline \multirow{2}{*}{ Sigmoid } & Before & 794 & 174.4608 & 0.0968525 & 402 & 161.9323 & 0.053553 \\
\cline { 2 - 8 } & After & 795 & 171.090 & 0.0099719 & 401 & 191.52642 & 0.009081 \\
\hline
\end{tabular}

Table (b.6): Application results of $\varepsilon$-SVR and $v$-SVR with $n=1500$

\begin{tabular}{|c|c|c|c|c|c|c|c|}
\hline \multirow{2}{*}{ Kernel } & Using & \multicolumn{3}{|c|}{$\varepsilon$-SVR=1500 } & \multicolumn{3}{c|}{$v$-SVR=1500 } \\
\cline { 3 - 8 } & PCA & No. SVR & RMSE & $R^{2}$ & No. SVR & RMSE & $R^{2}$ \\
\hline \multirow{2}{*}{ Linear } & Before & 1069 & 4.29137 & 0.4949637 & 603 & 4.32192 & 0.497399 \\
\cline { 2 - 8 } & After & 1080 & 4.30776 & 0.4872366 & 602 & 4.48947 & 0.4874544 \\
\hline \multirow{2}{*}{ Polynomial } & Before & 1076 & 4.979287 & 0.333197 & 605 & 5.064822 & 0.344293 \\
\cline { 2 - 8 } & After & 1084 & 4.82179 & 0.351424 & 603 & 5.12483 & 0.3504718 \\
\hline \multirow{2}{*}{ Radial } & Before & 1067 & 4.3989396 & 0.466456 & 623 & 4.501377 & 0.464170 \\
\cline { 2 - 8 } & After & 1079 & 4.3563 & 0.474433 & 618 & 4.5555559 & 0.463043 \\
\hline \multirow{2}{*}{ Sigmoid } & Before & 1199 & 314.6709 & 0.1760055 & 601 & 216.9770 & 0.0941660 \\
\cline { 2 - 8 } & After & 1190 & 205.508 & 0.0081285 & 602 & 80.25321 & 0.03242593 \\
\hline
\end{tabular}

Table (b.7):Application results of $\varepsilon$-SVR and v-SVR with $\mathrm{n}=1750$

\begin{tabular}{|c|c|c|c|c|c|c|c|}
\hline \multirow{2}{*}{ Kernel } & Using & \multicolumn{3}{|c|}{$\varepsilon$-SVR=1750 } & \multicolumn{3}{c|}{$v$-SVR=1750 } \\
\cline { 3 - 8 } & PCA & No. SVR & RMSE & $R^{2}$ & No. SVR & RMSE & $R^{2}$ \\
\hline \multirow{2}{*}{ Linear } & Before & 1080 & 5.12654 & 0.9296202 & 708 & 3.034645 & 0.9312248 \\
\cline { 2 - 8 } & After & 1027 & 3.94297 & 0.6098332 & 702 & 2.5561089 & 0.672139 \\
\hline \multirow{2}{*}{ Polynomial } & Before & 821 & 5.38031 & 0.9234148 & 711 & 3.0423366 & 0.9240209 \\
\cline { 2 - 8 } & After & 1083 & 4.234208 & 0.5439352 & 704 & 3.0099337 & 0.5328131 \\
\hline \multirow{2}{*}{ Radial } & Before & 1333 & 8.289448 & 0.9816035 & 724 & 3.13559 & 0.9827222 \\
\cline { 2 - 8 } & After & 769 & 6.7023958 & 0.8830206 & 709 & 2.974966 & 0.8840259 \\
\hline \multirow{2}{*}{ Sigmoid } & Before & 1381 & 577.73834 & 0.0121804 & 702 & 69.768746 & 0.00003041 \\
\cline { 2 - 8 } & After & 1394 & 496.99376 & 0.1055873 & 702 & 48.677263 & 0.09880482 \\
\hline
\end{tabular}


Table (b.8): Application results of $\varepsilon$-SVR and $v$-SVR with $n=2000$

\begin{tabular}{|c|c|c|c|c|c|c|c|}
\hline \multirow{2}{*}{ Kernel } & Using & \multicolumn{3}{|c|}{$\varepsilon$-SVR=2000 } & \multicolumn{3}{c|}{$v$-SVR=2000 } \\
\cline { 3 - 8 } & PCA & No. SVR & RMSE & $R^{2}$ & No. SVR & RMSE & $R^{2}$ \\
\hline \multirow{2}{*}{ Linear } & Before & 1080 & 1.0057917 & 0.9994493 & 805 & 1.0542278 & 0.99333 \\
\cline { 2 - 8 } & After & 1394 & 0.0539351 & 0.6137618 & 802 & 1.0873407 & 0.9999 \\
\hline \multirow{2}{*}{ Polynomial } & Before & 1260 & 1.449058 & 0.6674626 & 813 & 1.3706637 & 0.6756977 \\
\cline { 2 - 8 } & After & 1447 & 0.9712849 & 0.3253675 & 804 & 1.3895499 & 0.6756977 \\
\hline \multirow{2}{*}{ Radial } & Before & 1389 & 1.0335056 & 0.9883338 & 949 & 1.4172537 & 0.9933313 \\
\cline { 2 - 8 } & After & 1408 & 0.1752729 & 0.5916619 & 815 & 1.1007692 & 0.9933313 \\
\hline \multirow{2}{*}{ Sigmoid } & Before & 1598 & 109.76821 & 0.1382282 & 802 & 69.33145 & 0.06405554 \\
\cline { 2 - 8 } & After & 1591 & 80.915245 & 0.0192846 & 803 & 65.374727 & 0.06405554 \\
\hline
\end{tabular}

\section{Appendix (c)}

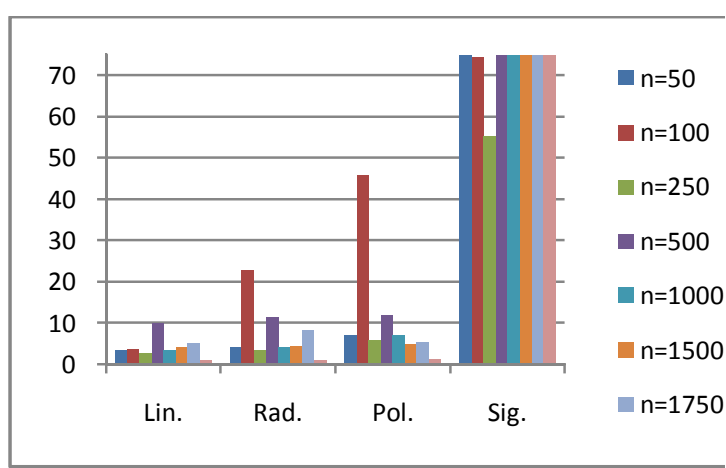

Fig. (c.1) Effect of PCA for $\varepsilon$-SVRat $n=1000$

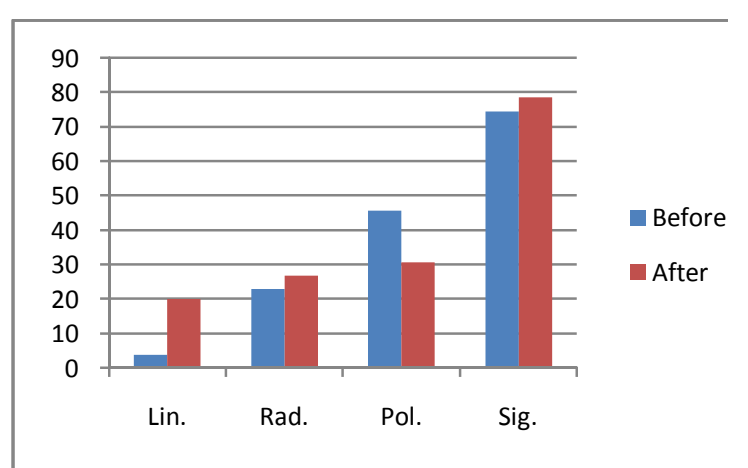

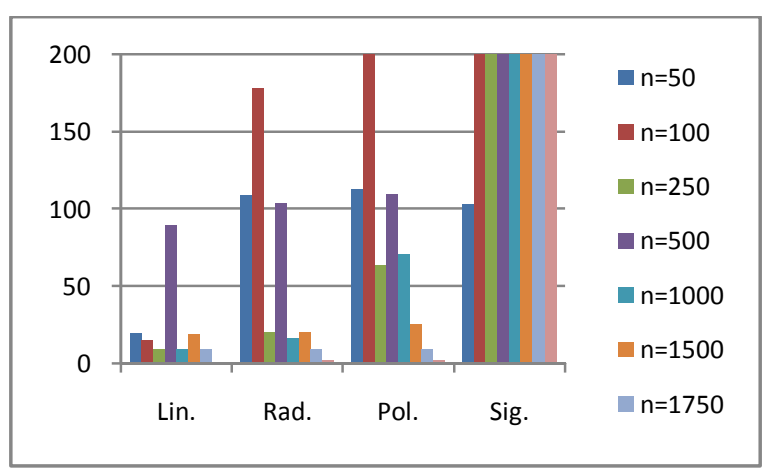

Fig. (c.2) Effect of PCA for $\varepsilon$-SVR at $n=1000$

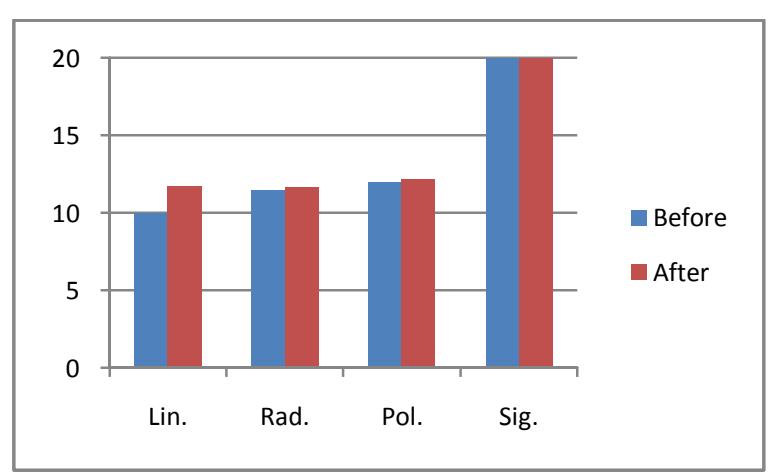

Fig. (c.3) Effect of PCA for $\varepsilon$-SVRat n=100Fig.(c.4) Effect of PCA for $\varepsilon$-SVR at $\mathrm{n}=500$ 


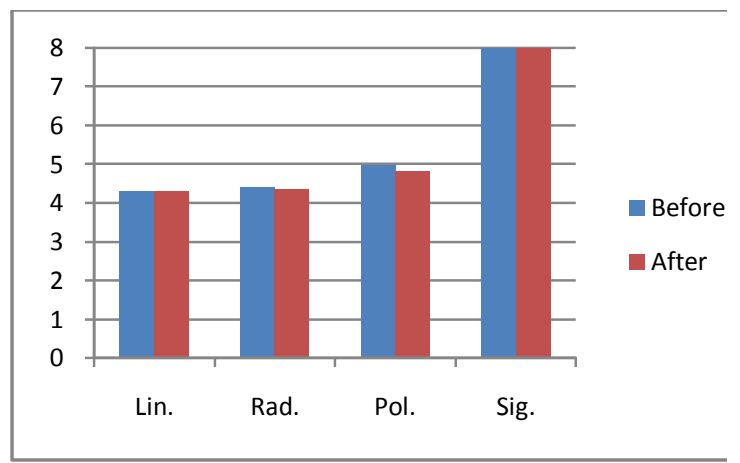

Fig. (c.5) Effect of PCA for $\varepsilon$-SVR at $n=$ 1500

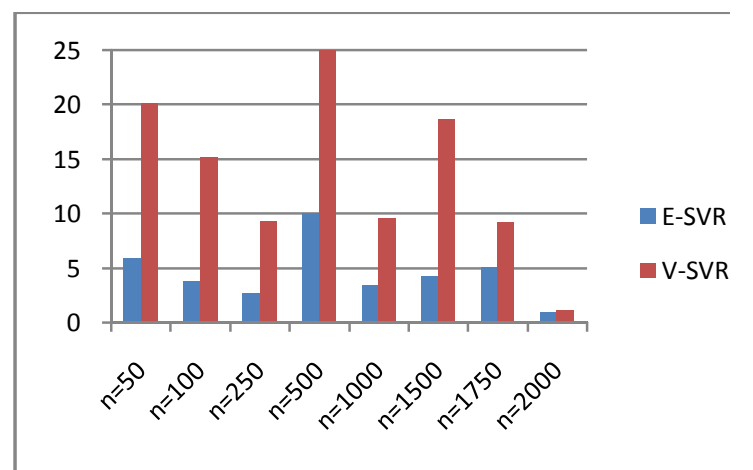

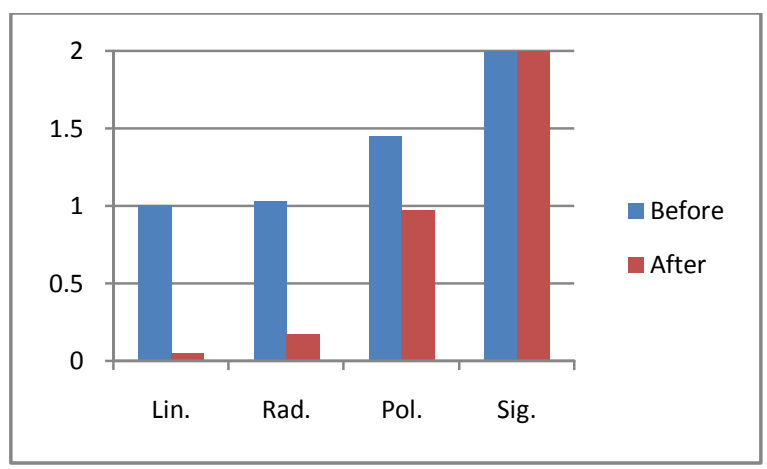

Fig.(c.6) Effect of PCA for $\varepsilon$-SVR at $n=$ 2000

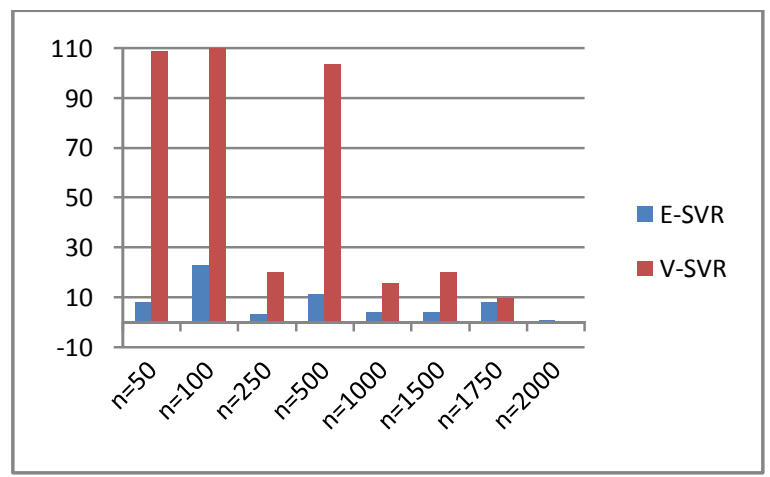

Fig.(c.9) effect of PCA for $v$-SVRat $n=50$ Fig. (c.8) effect of PCA forv-SVRat $n=250$

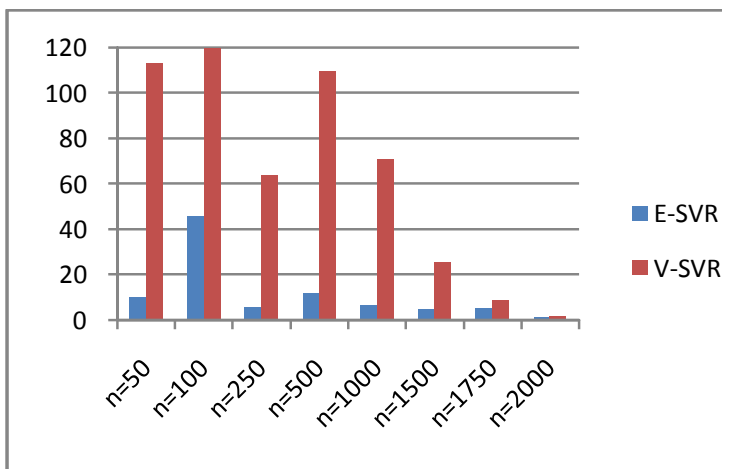

Fig. (c.9) effect of PCA for $v$-SVRat $n=1000$

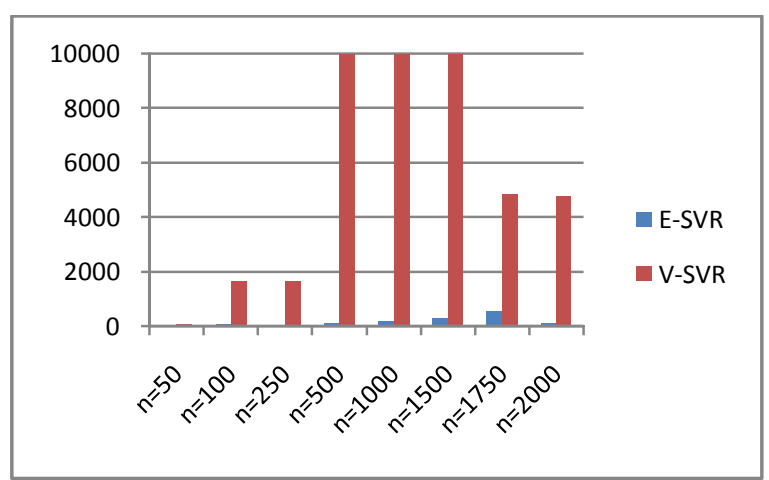

Fig. (c.10) effect of PCA for $v$-SVRat $n=1750$ 

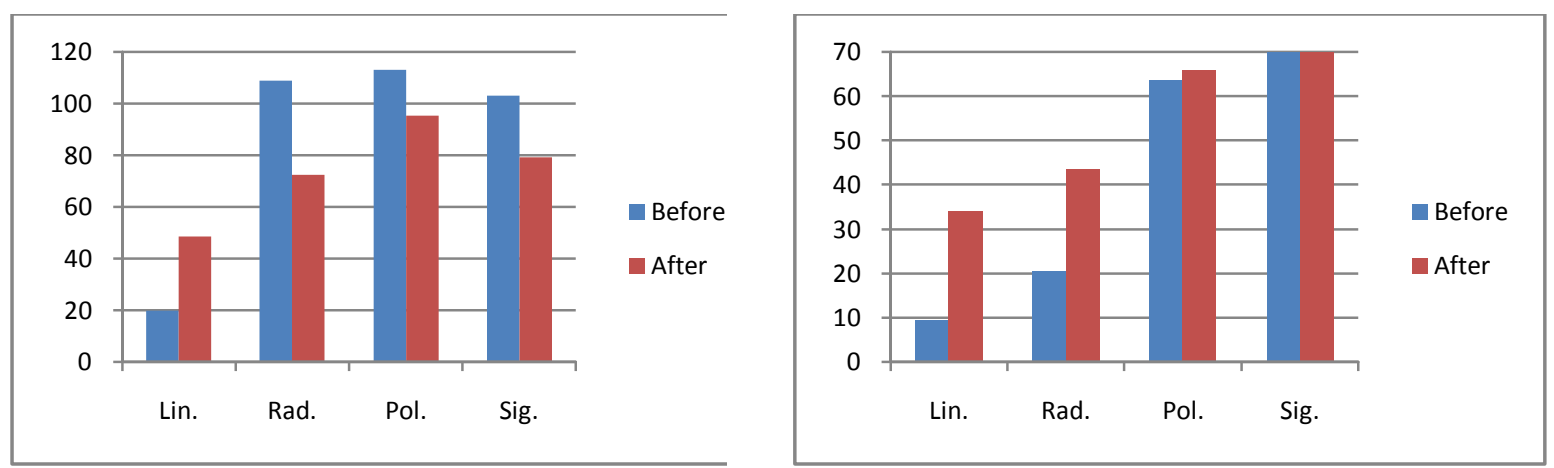

Fig.(c.11) Effect of SVR for Linear kernel Fig. (c.12) Effect of SVR for Radial kernel

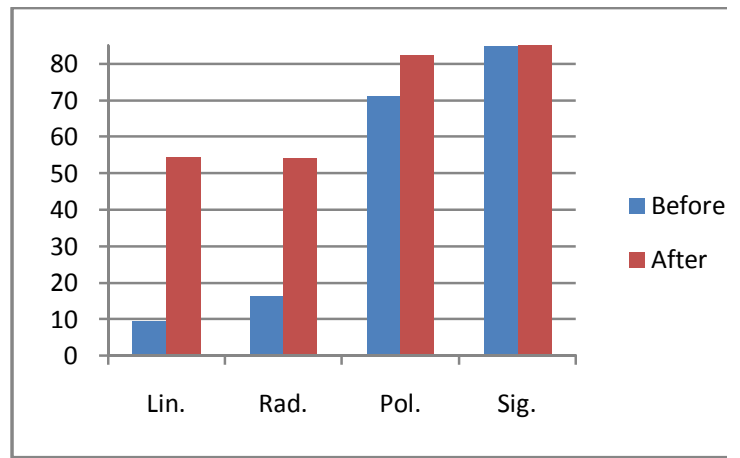

Fig. (c.13) Effect of SVR for Polynomial kernel

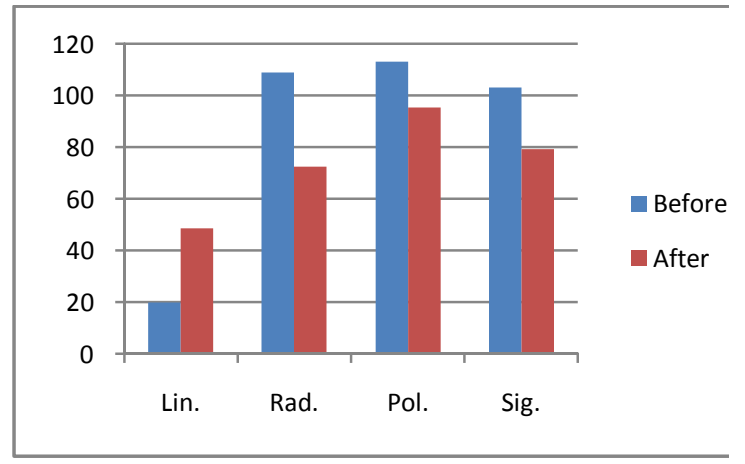

Fig. (c.15) $\varepsilon$-SVR before PCA

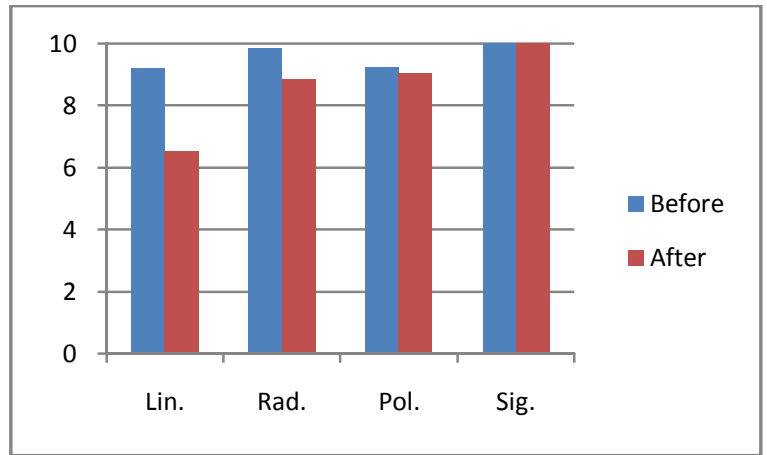

Fig. (c.14) Effect of SVR for Sigmoid kernel

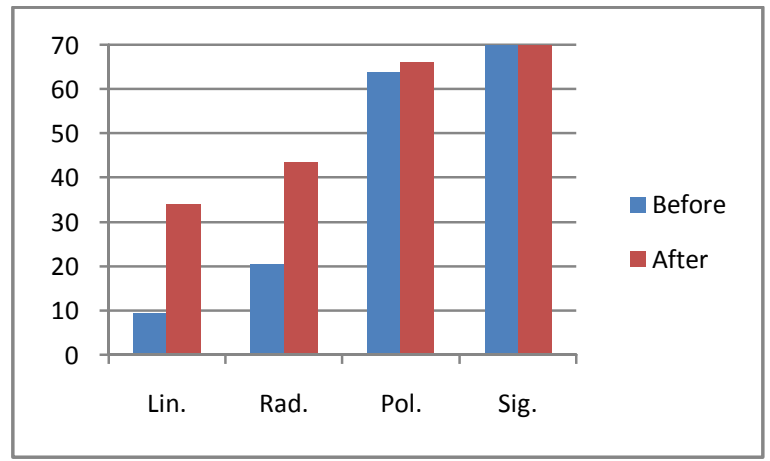

Fig. (c.16) $v$-SVR before PCA 